\title{
Analytical representations for the radial distribution functions of mixtures of adhesive spheres
}

\author{
CHRISTIAN TUTSCHKA ${ }^{1,2}$, GERHARD KAHL ${ }^{1,3 *}$ and ERWIN RIEGLER ${ }^{1}$ \\ ${ }^{1}$ Institut für Theoretische Physik, TU Wien, Wiedner Hauptstra ße 8-10, A-1040 \\ Vienna, Austria \\ ${ }^{2}$ Departamento de Matemáticas, Universidad Carlos III de Madrid, Avenida de la \\ Universidad 30, 28911-Leganés, Spain \\ ${ }^{3}$ CMS, TU Wien, Wiedner Hauptstraße 8-10, A-1040 Vienna, Austria
}

(Received 10 October 2001; revised version accepted 13 November 2001)

\begin{abstract}
A study has been made of the pole topology of the Laplace transforms of the pair distribution functions (PDFs) of a binary mixture of adhesive hard spheres (AHS) both for the PercusYevick equation and the mean spherical model (MSM). Expressions are given that describe how the distribution of the poles in the left half of the complex plane varies with the system parameters for the special case of the MSM for symmetric binary AHS mixtures. The locations of the poles closest to the imaginary axis are known to determine the asymptotic form of the PDFs, i.e. either exponentially monotonic or exponentially oscillatory decaying. As a byproduct of this inquiry analytical $r$ space representations of the PDFs are derived that allow their accurate and efficient determination over the entire $r$ range.
\end{abstract}

\section{Introduction}

The pair distribution function (PDF) $g(r)$ is one of the central quantities in the microscopic theory of classical fluids in thermal equilibrium. If we assume our system to be homogeneous and isotropic, this function measures the extent to which the bulk structure deviates from complete randomness. Due to its importance, particular effort has been devoted from both theoretical and experimental sides to determine this function as accurately as possible.

From the point of view of the quantitative theory many sophisticated concepts were developed to calculate the PDF (for an overview see, e.g. [1, 2]); nowadays, the methods available provide results for $g(r)$ that, within numerical accuracy, are in agreement with computer simulation data. This also holds for system states that are located near to the phase separation region and near criticality.

One of the first contributions to the qualitative theory of the PDF was that $g(r)$ tends (under certain conditions) towards unity for large $r$ (see, e.g. [3, 4]), proving thus the intuitive picture that (under the same conditions) two particles separated by a large distance $r$ are uncorrelated. It was soon afterwards found out that this convergence to unity, i.e. the asymptotic behaviour,

\footnotetext{
* Author for correspondence. e-mail: gkahl@tph.tuwien. ac.at
}

can be realized in only two ways [if all the singularities of $\hat{g}_{i j}(t)$ are poles (see below)]. Lebowitz and Percus [5] noted that for large distances a PDF has either an exponential decay or an exponentially damped oscillatory form, characterized either by a real or a complex decaying length. Systems that show one or other such behaviour are separated in the phase diagram by a line which in the literature is commonly referred to as the Fisher-Widom (FW) line [6]. Abraham and Kunz [7] established that this universal exponential decay of the PDF can be found for all continuous systems with finite range interactions, at least for low densities. In later work, Martynov [8] showed that, for the case of binary systems, the long distance behaviour of the partial PDFs is governed by only one decaying length. Detailed work in this field was carried out by Evans, Henderson and their respective colleagues [9-15]. In several studies they treated the universal asymptotic behaviour in a more systematic way: they investigated the location of the pole of the Laplace transform s of the PDFs of mixtures, $\hat{g}_{i j}(t)$, closest to the origin, that decides which of the two kinds of asymptotic behaviour is realized. Instead of searching the poles of $\hat{g}_{i j}(t)$ in the complex $t$ plane, Evans and coworkers [9-15] investigated the Fourier transforms of the PDFs in the complex $q$ plane (from the elementary relation between Fourier and Laplace transforms it follows that these two sets of poles are related via a rotation by $\pi / 2$ ). In addition they could show that the universal exponential 
convergence of the PDFs to their asymptotic value is not only present for the case of a uniform fluid, but also for density profiles of the corresponding non-uniform system.

In previous treatments we have considered the distribution of the poles of the $\hat{g}_{i j}(t)$ in the left half $(\mathrm{LH})$ of the complex plane for binary hard sphere (HS) [16] and binary hard sphere Yukawa (HSY) mixtures [17]. Based on the Percus-Yevick (PY) equation for HS and on the mean spherical model (MSM) for HSY, closed analytical expressions can be derived for the $\hat{g}_{i j}(t)$ of these systems, facilitating all subsequent calculations substantially. These functions have an infinite number of poles in the LH of the complex $t$ plane. The locations of the poles (referred to as the pole topology) show characteristic patterns: in both cases we observe that the poles are arranged in two branches that, for large parts of the parameter space, are clearly separated. However, we have observed a characteristic difference between these two systems: HS mixtures that, within the PY equation, do not phase separate, have one pole on the negative real axis, whereas, by contrast, HSY mixtures that, within the MSM, do phase separate, have two poles on the negative real axis. We surmise that a relation exists between the distribution of poles on the negative real axis and the existence of phase transitions in a system. Restricting to the poles closest to the imaginary axis, we observed for the case of binary HS mixtures only exponentially damped oscillatory behaviour (cf. also [7] for the one-component case of dilute HS), while for the HSY mixture both cases, i.e. real or complex decay lengths, were encountered. Although qualitative predictions could be made on how the parameters influence the location of the branches in the $t$ plane [17], the number of parameters for a binary HSY mixture was too large to establish a theory of the pole topology of the $\hat{g}_{i j}(t)$.

In the present contribution we elucidate this point by examining an analytical solvable system with a parameter space of considerably smaller dimensions. To this end we choose a system that, with respect to the complexity of its interaction, is between HS and HSY systems: we consider binary mixtures of adhesive hard spheres (AHSs) [18], within both the PY equation and the MSM. They represent models for our aims: first, the PDFs of the PY equation [19] and of the MSM [20] for mixtures of AHSs can be calculated analytically; second, with respect to HS mixtures on the one hand these represent the most simple extension with an attractive interaction, being thus able to undergo a phase transition, and with respect to HSY mixtures on the other hand, their interactions are characterized by only one set of parameters, i.e. the stickiness, yielding thus the desired reduction in the dimensionality of the parameter space.
Applying the same approach that we have used to determine the pole topology for HSY mixtures [17], we have treated the pole topology of the MSM for a symmetric binary mixture of AHSs. We have restricted ourselves to the MSM in an effort to obtain as simple expressions as possible: the MSM provides a density independent solution for the parameters of the structure functions [20], while the PY equation leads to a more complicated, i.e. density dependent solution [19]. In the present context this does not represent a serious limitation, since we are interested in only a qualitative understanding of the pole topology of the PDFs. Indeed, for the MSM we are able to give expressions showing how the location of the branches depends on the system parameters.

As a by-product of our analysis we have adapted two analytical representations that can be combined into an accurate and efficient $r$ space method to calculate PDFs over the entire $r$ range: the expressions used in these techniques, commonly referred to as the shell representation (SR) and the asymptotic representation (AR), are also presented in this contribution.

\section{Basic solutions of the model}

A binary AHS mixture is characterized by the number density $\rho$ and the concentrations $c_{i}$ of species $i(i=1,2)$, with partial number densities $\rho_{i}=c_{i} \rho$ and partial packing fractions $\eta_{i}=\pi / 6 \rho_{i}$. The set of interactions is defined by $[18,19]$

$$
\begin{aligned}
& \beta \Phi_{i j}(x)= \\
& \lim _{\epsilon \rightarrow \infty} \begin{cases}\infty & r<R_{i j}, \\
-\Theta\left(r-R_{i j}\right) \log \left[\frac{\gamma_{i j} R_{i j}}{\epsilon}\right] \Theta\left(R_{i j}+\epsilon-r\right) & R_{i j} \leq r,\end{cases}
\end{aligned}
$$

where $\Theta(r)$ is the usual Heaviside step function and the parameters $\gamma_{i j} \in \mathbb{R}_{+}^{2 \times 2}$ represent the stickiness between particles $i$ and $j$. The $R_{i i}=R_{i}$ are the hard core diameters, and we furthermore define $R_{i j}=\frac{1}{2}\left(R_{i}+R_{j}\right)$, $S_{i j}=\frac{1}{2}\left(R_{i}-R_{j}\right)$, and assume $S_{i j} \geq 0 . \quad \beta=1 / k_{\mathrm{B}} T, k_{\mathrm{B}}$ being the Boltzmann constant and $T$ the temperature.

We investigate the PDFs, $g_{i j}(r)$, of equation (1) from the Ornstein-Zernike (OZ) equations

$h_{i j}(r)=c_{i j}(r)+\sum_{k=1,2} \rho_{k} \int \mathrm{d} \mathbf{r}^{\prime} c_{i k}\left(r^{\prime}\right) h_{k j}\left(\left|\mathbf{r}-\mathbf{r}^{\prime}\right|\right) \quad i, j=1,2$,

for the PY equation and the MSM. $h_{i j}(r)=\left[g_{i j}(r)-1\right]$ and the $c_{i j}(r)$ are the total and direct correlation functions. To obtain analytical solutions of the $\mathrm{OZ}$ equations with the above closures for the present system we 
have applied the Wiener-Hopf (WH) factorization [21] that transforms these equations into two sets of coupled integral equations (WH equations) for the so-called factor functions $Q_{i j}(r)$ (for details, cf. [21]). Note that in this contribution we use the convention of [22].

Finally, one finds for $Q_{i j}(r)$ both for the PY equation and the MSM [19, 20] (note the 'translation rule' between the factor functions $Q_{i j}$ used by Blum and Høye [22] and those $q_{i j}$ used by Baxter [21], Perram and Smith [19], and Tutschka and Kahl [20]: $Q_{j i}=Q_{i j}^{\mathrm{T}}=2 \pi q_{i j}$.

$\frac{1}{2 \pi} Q_{i j}(r)$

$=\left\{\begin{array}{c}\frac{a_{j}}{2}\left(r-R_{i j}\right)^{2}+\left(b_{j}+a_{j} R_{i j}\right)\left(r-R_{i j}\right) \\ +\lambda_{i j} R_{i j}^{2} \\ 0\end{array}\right.$

$S_{j i}<r<R_{i j}$, elsewhere,

with

$$
\begin{aligned}
a_{i} & =\frac{1-12 \zeta_{i}}{1-\xi_{3}}+\frac{3 R_{i} \xi_{2}}{\left(1-\xi_{3}\right)^{2}}, \\
b_{i} & =-\frac{3}{2} \frac{R_{i}^{2} \xi_{2}}{\left(1-\xi_{3}\right)^{2}}+\frac{6 \zeta_{i} R_{i}}{1-\xi_{3}} \quad i=1,2,
\end{aligned}
$$

and

$$
\xi_{n}=\frac{\pi}{6} \sum_{i=1,2} \rho_{i} R_{i}^{n}, \quad \zeta_{i}=\frac{\pi}{6} \sum_{j=1,2} \rho_{j} \lambda_{i j} R_{i j}^{2} R_{j},
$$

while the explicit dependence of $\lambda_{i j}$ on the density and stickiness parameters (see below) is different for the PY and the MSM cases.

The PY equation leads to the set of four coupled quadratic equations [19]

$$
\begin{aligned}
\lambda_{i j}^{\mathrm{PY}} R_{i j}= & \gamma_{i j}\left[\frac{R_{i j}}{1-\xi_{3}}+\frac{3 R_{i} R_{j} \xi_{2}}{2\left(1-\xi_{3}\right)^{2}}-6 \frac{\zeta_{i} R_{j}+\zeta_{j} R_{i}}{1-\xi_{3}}\right. \\
& \left.+2 \pi \sum_{k=1,2} \rho_{k} \lambda_{i k}^{\mathrm{PY}} R_{i k}^{2} \lambda_{k j}^{\mathrm{PY}} R_{k j}^{2}\right] .
\end{aligned}
$$

These equations are nonlinear and the $\lambda_{i j}^{\mathrm{PY}}$ are explicitly density dependent. For our case of a binary mixture one obtains eight solutions, and the physical solution is picked out according to the following criteria: the $\lambda_{i j}^{\mathrm{PY}}$ have to be real and positive, symmetric in $i$ and $j$, and have to fulfil $\lim _{\rho \rightarrow 0} \lambda_{i j}^{\mathrm{PY}}=\gamma_{i j}$.

To obtain the MSM for AHSs one considers a special limiting case $[23,20]$ of an HSY interaction: using the analytical WH solution of the $\mathrm{OZ}$ equations with Yukawa closure one examines this solution in the limit where the interaction becomes (in a suitable manner) both infinitely strong and infinitely short ranged, thus representing the surface adhesion of AHSs. Finally, one arrives at $[20,23]$

$$
\lambda_{i j}^{\mathrm{MSM}}=\gamma_{i j} .
$$

Note that this result agrees with the above mentioned low density limit of the PY solution.

In passing, we remark that the solution of the original $\mathrm{OZ}$ equation is equivalent to the solution obtained via the $\mathrm{WH}$ factorization, provided that $[21,24]$

$$
\begin{array}{r}
\Delta(k)=\operatorname{det}\left[\delta_{i j}-\sqrt{\rho_{i} \rho_{j}} \int_{S_{j i}}^{R_{i j}} \mathrm{e}^{i k r} Q_{i j}(r) \mathrm{d} r\right]>0 \\
\text { for all } \operatorname{Im}(k) \geq 0 .
\end{array}
$$

A necessary condition for the above criterion is the requirement that $\Delta(0)>0[21]$.

\section{Shell and asymptotic representations}

\subsection{General expressions}

The PDFs can now be calculated from the $Q_{i j}(r)$ via the WH equations; to this end we define $\hat{g}_{i j}(t)$ and $\hat{Q}_{i j}(t)$

$$
\hat{g}_{i j}(t)=\int_{0}^{\infty} \mathrm{d} r \mathrm{e}^{-t r} r g_{i j}(r), \quad \hat{Q}_{i j}(t)=\int_{S_{j i}}^{R_{i j}} \mathrm{~d} r \mathrm{e}^{-t r} Q_{i j}(r) .
$$

Evaluating the $\hat{Q}_{i j}(t)$ from equation (3) we find

$$
\hat{Q}_{i j}(t)=\frac{1}{2 t^{3}} \mathrm{e}^{-t R_{i j}}\left\{f_{i j}^{0}(t)+\mathrm{e}^{t R_{i}}\left[-f_{i j}^{0}(t)+f_{i j}^{1}(t)\right]\right\},
$$

where we have introduced the $f_{i j}^{\alpha}(t), i, j=1,2$,

$$
\begin{aligned}
& f_{i j}^{0}(t)=2 \pi\left[-2 t^{2} R_{i j}^{2} \lambda_{i j}-2 t\left(a_{j} R_{i j}+b_{j}\right)-2 a_{j}\right], \\
& f_{i j}^{1}(t)=2 \pi\left[-t^{2}\left(a_{j} R_{i} R_{j}+2 b_{j} R_{i}\right)-2 t a_{j} R_{i}\right] .
\end{aligned}
$$

The WH formalism gives the following set of equations for $\hat{g}_{i j}(t)$ and $\hat{Q}_{i j}(t)$ :

$$
\sum_{l=1,2} 2 \pi \hat{g}_{i l}(t)\left[\delta_{l j}-\rho_{l} \hat{Q}_{l j}(t)\right]=\frac{1}{t^{2}} \mathrm{e}^{-t R_{i j}}\left[-\frac{1}{2} f_{i j}^{0}(t)\right] .
$$

For details we refer the reader either to [22] (HSY mixture) or to [20] ( $N$-component mixture of AHSs).

We define

$$
\begin{aligned}
L_{0}(t)= & \frac{1}{t^{2}} \rho_{1} \rho_{2}\left[f_{11}^{0}(t) f_{22}^{0}(t)-f_{12}^{0}(t) f_{21}^{0}(t)\right] \\
L_{1}(t)= & \frac{1}{t^{2}} \rho_{2}\left\{2 t^{3} f_{22}^{0}(t)-\rho_{1}\left[f_{22}^{0}(t) f_{11}^{1}(t)-f_{21}^{0}(t) f_{12}^{1}(t)\right]\right\} \\
& +L_{0}(t)
\end{aligned}
$$




$$
\begin{aligned}
L_{2}(t)= & \frac{1}{t^{2}} \rho_{1}\left\{2 t^{3} f_{11}^{0}(t)-\rho_{2}\left[f_{11}^{0}(t) f_{22}^{1}(t)-f_{12}^{0}(t) f_{21}^{1}(t)\right]\right\} \\
+L_{0}(t) & \\
L_{3}(t)= & \frac{1}{2 \pi} \frac{1}{t^{3}}\left\{-t^{3}\left[f_{12}^{0}(t)+f_{21}^{0}(t)\right]\right. \\
& +\frac{1}{2} \rho_{1}\left[f_{11}^{1}(t) f_{12}^{0}(t)-f_{11}^{0}(t) f_{12}^{1}(t)\right] \\
& \left.+\frac{1}{2} \rho_{2}\left[f_{22}^{1}(t) f_{21}^{0}(t)-f_{22}^{0}(t) f_{21}^{1}(t)\right]\right\} \\
S(t)= & \frac{1}{t^{2}}\left\{4 t^{6}-2 t^{3}\left[\rho_{1} f_{11}^{1}(t)+\rho_{2} f_{22}^{1}(t)\right]\right. \\
& \left.+\rho_{1} \rho_{2}\left[f_{11}^{1}(t) f_{22}^{1}(t)-f_{12}^{1}(t) f_{21}^{1}(t)\right]\right\} \\
& +L_{1}(t)+L_{2}(t)-L_{0}(t), \\
D(t)=L_{0}(t) & -L_{1}(t) \mathrm{e}^{t R_{1}}-L_{2}(t) \mathrm{e}^{t R_{2}}+S(t) \mathrm{e}^{t\left(R_{1}+R_{2}\right)} .
\end{aligned}
$$

Note that the $L_{i}(t)(i=0, \ldots, 3)$ and $S(t)$, despite of the prefactors proportional to $t^{-n}$, are indeed polynomials in $t$ (similar to HS and HSY mixtures): the $L_{i}(t)$ $(i=0, \ldots, 3)$ are polynomials of degrees $2,3,3$, and 2 , and $S(t)$ is of degree 4 . One should also point out that the following relation holds

$$
D(t)=4 t^{4} \mathrm{e}^{t\left(R_{1}+R_{2}\right)} \Delta(i t) .
$$

With the above definitions we finally arrive at the following compact expressions for $\hat{g}_{i j}(t)$ (symmetrizing explicitly the expression for $\hat{g}_{12}(t)$ )

$$
\begin{aligned}
& \hat{g}_{11}(t)=\frac{1}{12 \eta_{1}} \frac{t}{D(t)}\left[L_{0}(t)-\mathrm{e}^{t R_{2}} L_{2}(t)\right]=\frac{N_{11}(t)}{D(t)}, \\
& \hat{g}_{12}(t)=\frac{t^{2}}{D(t)} \mathrm{e}^{t R_{12}} L_{3}(t)=\frac{N_{12}(t)}{D(t)}, \\
& \hat{g}_{22}(t)=\frac{1}{12 \eta_{2}} \frac{t}{D(t)}\left[L_{0}(t)-\mathrm{e}^{t R_{1}} L_{1}(t)\right]=\frac{N_{22}(t)}{D(t)} .
\end{aligned}
$$

Here some comments are in order. (i) The form of these expressions for $\hat{g}_{i j}(t)$ is identical with that derived for the PY equation of binary HS mixtures [16] and for the MSM of binary HSY mixtures [17]. Indeed, the fact that $\hat{g}_{i j}(t)$ can be represented in such a form (if $Q_{i j}(r)$ can be expressed in terms of analytical functions) is a consequence of the structure of the underlying WH equations. Another example of this common algebraic structure is provided by the MSM solution for systems of charged HSs [25]. (ii) The well known delta function singularity at $r=R_{i j}$ of the PDFs of the PY equation and the MSM for AHSs [26] is reproduced within the WH formalism from one of the WH equations [21],

$$
\begin{aligned}
2 \pi r h_{i j}(|r|)= & -Q_{i j}^{\prime}(r) \\
& +2 \pi \sum_{k=1,2} \rho_{k} \int_{S_{j k}}^{R_{k j}} \mathrm{~d} t(r-t) h_{i k}(|r-t|) Q_{k j}(t) \\
S_{j i}<r, & (23)
\end{aligned}
$$

and the discontinuity of $Q_{i j}(r)$ at $r=R_{i j}$, equation (3). (iii) As an immediate consequence of this singularity and of equations (3) and (26), a finite step in the PDFs is found at $r=R_{i j}+R_{k}, k=1,2$; one obtains explicitly

$$
\begin{aligned}
& {\left[r h_{i j}(r)\right]_{r=R_{i j}+R_{k}^{-}}-\left[r h_{i j}(r)\right]_{r=R_{i j}+R_{k}^{+}}} \\
& \quad=-2 \pi \rho_{k} \lambda_{i k} R_{i k}^{2} \lambda_{k j} R_{k j}^{2}, \quad k=1,2 .
\end{aligned}
$$

With $\hat{g}_{i j}(t)$ being transformed into expressions (20)(22) we can apply the SR and the AR, which were presented in detail in [16] (see also [17] for the notation used here). Since the SR evaluates $g_{i j}(r)$ via an application of the residue theorem, the PDFs are obtained only in open intervals, e.g. $\left\{r \mid R_{k}>r-\left(\alpha_{1} R_{1}+\alpha_{2} R_{2}\right)>0\right\}$, $k=1,2$, with $\alpha_{1}, \alpha_{2}$ being integers or half-integers. In particular, the SR gives only the left and right limits of the PDFs at $r=R_{i j}$, i.e. $g_{i j}\left(R_{i j}^{-}\right)$and $g_{i j}\left(R_{i j}^{+}\right)$, but does not lead to the delta function singularity at $r=R_{i j}$ (the residue theorem is not applicable at $r=R_{i j}$ due to the lack of a suitable contour). Neither, for the same reason, does the AR provide this singularity at $r=R_{i j}$. However, this methodological limitation is not a serious one, since the singular part of the PDFs is at hand in $r$ space directly from equations (3) and (23). The expressions given in the two following subsections for the PDFs can be used to calculate the non-singular parts of $g_{i j}(r)$.

\subsection{Shell representation}

As outlined in [16], we expand the denominator $D(t)$ in equations (20)-(22) for sufficiently large $\operatorname{Re}(t)$, so that $D(t)$ becomes a power series of $S(t)$,

$$
\frac{1}{D(t)}=\frac{\exp \left[-t\left(R_{1}+R_{2}\right)\right]}{S(t)} \sum_{n=0}^{\infty}\left[\frac{I(t)}{S(t)}\right]^{n},
$$

with $I(t)$ given in [17], equation (23). Hence, one arrives at the following expressions for the inverse Laplace transform of $g_{11}(r)$,

$$
\begin{aligned}
& r g_{11}(r)=\frac{1}{12 \eta_{1}} \sum_{n=0}^{\infty} \frac{1}{2 \pi i} \\
& \times \int_{\mathcal{C}} \frac{t\left[L_{0}(t)-L_{2}(t) \mathrm{e}^{t R_{2}}\right][I(t)]^{n} \exp \left\{t\left(r-\left[R_{1}+R_{2}\right]\right)\right\}}{[S(t)]^{n+1}} \mathrm{~d} t
\end{aligned}
$$




$$
\begin{aligned}
= & \frac{1}{12 \eta_{1}} \sum_{n=0}^{\infty} \frac{1}{2 \pi i} \\
& \times \int_{\mathcal{C}}\left[\frac{1}{[S(t)]^{n+1}} \sum_{\alpha_{1}, \alpha_{2}} t Q_{n ; \alpha_{1} \alpha_{2}}(t)\right. \\
& \left.\times \exp \left\{t\left[r-\left(\alpha_{1} R_{1}+\alpha_{2} R_{2}\right)\right]\right\}\right] \mathrm{d} t, \\
\alpha_{1} & =1, \ldots, n+1, \quad \alpha_{2}=n+1-\alpha_{1}, \ldots, n+1,
\end{aligned}
$$

where $\mathcal{C}$ is a suitable contour in the complex $t$ plane and the $Q_{n ; \alpha_{1}, \alpha_{2}}(t)$, being products of $L_{0}(t), L_{1}(t)$, and $L_{2}(t)$, are formally identical to the corresponding expressions of the HS case which can be found in [16], appendix.

Evaluation in expression (26) is carried out via the residue theorem, where $\mathcal{C}$ has to be taken along a line in the right half $(\mathrm{RH})$ of the complex $t$ plane, parallel to the imaginary axis and to the right of all the poles of the integrand, i.e. the four zeros of $S(t)$, denoted by $t_{i}$. If $r$ is now either smaller or larger than $\alpha_{1} R_{1}+\alpha_{2} R_{2}$, the path parallel to the imaginary axis has to be closed either by a semicircle in the $\mathrm{RH}$ or in the $\mathrm{LH}$ of the plane. In the first case, all the poles of the integrand will lie outside the area encircled by $\mathcal{C}$, and hence the value of this term in the series (26) of $g_{11}(r)$ will be zero. In the other case, all the poles of the integrand lie within the enclosed area, and the value of the integral is then given by the residues $R_{n ; \alpha_{1} \alpha_{2}}^{i}$ of the integrand; the explicit expressions (for simple multiplicity of all the roots of $S(t)$ ) are found in [16], equation (19), replacing simply the HS expressions for the $Q_{n ; \alpha_{1} \alpha_{2}}(t)$ by those for the AHS case.

Thus, on introducing the shell PDFs, $g_{n ; \alpha_{1}, \alpha_{2}}^{11}(r)$, with their support being the subshells $S_{n ; \alpha_{1} \alpha_{2}}$, defined via

$$
\begin{gathered}
S_{n ; \alpha_{1} \alpha_{2}}=\left\{r \mid r>\alpha_{1} R_{1}+\alpha_{2} R_{2}\right\}, \\
1 \leq \alpha_{1} \leq n+1, \quad n+1-\alpha_{1} \leq \alpha_{2} \leq n+1,
\end{gathered}
$$

one finally obtains for $g_{11}(r)$

$$
\begin{aligned}
r g_{11}(r)= & \sum_{n=0}^{\infty} \sum_{\alpha_{1}, \alpha_{2}} r g_{n ; \alpha_{1} \alpha_{2}}^{11}(r) \Theta\left[r-\left(\alpha_{1} R_{1}+\alpha_{2} R_{2}\right)\right], \\
r g_{n ; \alpha_{1} \alpha_{2}}^{11}(r)= & \frac{1}{12 \eta_{1}} \frac{1}{n !} \sum_{i=1}^{4} a_{n}^{i} \exp \left\{t_{i}\left[r-\left(\alpha_{1} R_{1}+\alpha_{2} R_{2}\right)\right]\right\} \\
& \times \sum_{j=0}^{n} b_{n ; \alpha_{1} \alpha_{2}}^{j, i}\left[r-\left(\alpha_{1} R_{1}+\alpha_{2} R_{2}\right)\right]^{j}, \\
1 \leq & \alpha_{1} \leq n+1, \quad n+1-\alpha_{1} \leq \alpha_{2} \leq n+1 .
\end{aligned}
$$

The $a_{n}^{i}$ are given by

$$
a_{n}^{i}=\frac{1}{n !} \frac{1}{\left[S^{\prime}\left(t_{i}\right)\right]^{n+1}}
$$

correcting thus a typo in [16] equation (A3); the other coefficients, i.e. $b_{n ; \alpha_{1} \alpha_{2}}^{j, i}$, can be taken directly from [16], equations (A4)-(A10) (with the HS polynomials replaced by the corresponding AHS polynomials). The expressions presented there allow the computation of the PDFs for distances up to $\alpha_{1} R_{1}+\alpha_{2} R_{2}, \alpha_{1}+\alpha_{2}=7$.

Likewise, the results for $g_{12}(r)$ and $g_{22}(r)$ may be derived; they are not presented here since their forms are identical to those of the HSY case [17].

\subsection{Asymptotic representation}

The AR has been discussed in detail in [16], and therefore we restrict ourselves in the present contribution only to the essential idea and the most relevant properties. The AR starts with the inverse Laplace transform of $\hat{g}_{i j}(t)$, i.e.

$$
r g_{i j}(r)=\frac{1}{2 \pi \mathrm{i}} \int_{\delta-\mathrm{i} \infty}^{\delta+\mathrm{i} \infty} \frac{N_{i j}(t)}{D(t)} \mathrm{e}^{t r} \mathrm{~d} t,
$$

$\delta$ being an arbitrary positive number. For $r<R_{i j}$, this integral is evaluated by using a contour $\mathcal{C}$ in the $\mathrm{RH}$ of the complex plane, yielding $g_{i j}(r)=0$; for $r>R_{i j}, \mathcal{C}$ is closed in the LH of the plane, where $D(t)$ has an infinite number of roots $t_{k}$, and the value of $g_{i j}(r)$ is therefore given by the infinite sum over the residues of $\hat{g}_{i j}(t)$ at $t=t_{k}$. The essential idea of the AR is to evaluate only the dominant part thereof [16]: this is accomplished by the ordering of the poles $t_{k}$ according to their negative real parts (pairs of conjugate poles count as one), starting with a pole of order 2 at $t=0$; as can be shown, the latter is contributing a term 1 to the PDFs [16]. Although up to now there is no proof, there is evidence (in particular for the systems considered in $\S 4$ [27]) that all other poles $t_{k}$ are simple. Hence one arrives at [16]

$$
g_{i j}(r)=1+\sum_{k=2}^{\infty} \xi\left(t_{k}\right) \operatorname{Re}\left(\frac{N_{i j}\left(t_{k}\right)}{D^{\prime}\left(t_{k}\right)} \frac{\mathrm{e}^{t_{k} r}}{r}\right), \quad r>R_{i j},
$$

with $\xi(t)=1$ for $t \in \mathbb{R}$ and $\xi(t)=2$ for $t \in \mathbb{C}$.

We now list some properties and consequences of the $\mathrm{AR}$, as given by equation (31) (cf. [16, 17]). (i) Except for $r$ values where the PDFs have finite step discontinuities (Gibbs phenomenon), the AR (31) converges very quickly to $g_{i j}(r)$. (ii) Hence, the combination of the SR near contact and for intermediate $r$ values (the finite step discontinuities are located at $r=R_{i j}+R_{k}, k=1,2$ ) with the AR for large $r$ values constitutes an accurate and efficient analytical $r$ space representation of the PDFs over the whole $r$ range $[16,17]$. (iii) Note also that the formalism of the AR contains the results of Lebowitz and Percus [5] and of Martynov [8] in a natural way. (a) The PDF tends to its asymptotic value, either exponentially monotonic $\left(t_{1} \in \mathbb{R}\right)$ or exponentially oscil- 
latory $\left(t_{1} \in \mathbb{C}\right)$; the two regimes in phase space where these two different types of behaviour are encountered are separated by the Fisher-Widom line [6]. (b) All three PDFs, $g_{i j}(r)$, due to their common denominator in (31), have the same asymptotic behaviour.

\section{Pole topology}

We now present results for the pole topology for a few selected systems; their system parameters are listed in table 1. To further reduce the number of parameters we restrict ourselves to a special binary mixture which in the literature is commonly referred to as a symmetric binary mixture. It is characterized by equal size particles (we put the diameters $R_{i}=1$ ) and the following relations between the stickiness parameters $\gamma_{i j}$ :

$$
\gamma_{22}=\gamma_{11}, \quad \gamma_{21}=\gamma_{12}, \quad \gamma_{12}=\mu \gamma_{11}
$$

We are thus left with the following four system parameters: the total packing fraction $\eta=\eta_{1}+\eta_{2}$, $c_{1}=\left(1-c_{2}\right), \gamma_{11}$, and $\mu$.

We start with deriving the expressions that describe the location of the two pole branches in the complex $t$ plane. The poles are determined from the equation $D(t)=0$, which can be rewritten as a quadratic equation for $\mathrm{e}^{-t}$, i.e.

$$
\sum_{k=0}^{2} c_{k}(t) \mathrm{e}^{-k t}=0
$$

with suitably chosen coefficients $c_{k}(t)$. Let $\chi_{1,2}(t)$ be the two roots of (33) and define $p(t)=\frac{1}{2}\left(\chi_{1}(t)+\chi_{2}(t)\right)$ as well as $q(t)=\frac{1}{2}\left(\chi_{1}(t)-\chi_{2}(t)\right)$. Then the two branches of poles, denoted by $t_{k}^{(1)}$ and $t_{k}^{(2)}$ (the latter being the one closer to the imaginary axis), are found to be

$$
\begin{aligned}
& \mathrm{e}^{-t_{k}^{(1)}}=\omega_{1}\left(t_{k}^{(1)}\right), \\
& \mathrm{e}^{-t_{k}^{(2)}}=\omega_{2}\left(t_{k}^{(2)}\right),
\end{aligned}
$$

with

$$
\begin{aligned}
& \omega_{1}(t)=p(t)+\operatorname{sign}[\operatorname{Re}(p(t))] \operatorname{sign}[\operatorname{Re}(q(t))] q(t), \\
& \omega_{2}(t)=p(t)-\operatorname{sign}[\operatorname{Re}(p(t))] \operatorname{sign}[\operatorname{Re}(q(t))] q(t) .
\end{aligned}
$$

The sign functions are introduced in an effort to keep the branches ordered. These expressions as such are not very instructive since the explicit dependence of equations (36) and (37) on the system parameters is quite involved. Therefore we have analysed the leading order asymptotic behaviour of equations (36) and (37) for $|t| \rightarrow \infty$ in the LH of the $t$ plane and obtained

$$
\omega_{1}(t) \asymp-\frac{t}{24 \eta \gamma_{11} c_{1} c_{2}\left(1-\mu^{2}\right)}\left(1+\sqrt{1-4 c_{1} c_{2}\left(1-\mu^{2}\right)}\right),
$$

$$
\omega_{2}(t) \asymp-\frac{t}{24 \eta \gamma_{11} c_{1} c_{2}\left(1-\mu^{2}\right)}\left(1-\sqrt{1-4 c_{1} c_{2}\left(1-\mu^{2}\right)}\right) .
$$

It follows from the form of expressions (38) and (39) that, at least asymptotically, the two branches never intersect (except for the trivial case of $c_{1}=c_{2}$ and $\mu=0)$. Furthermore, three facts can be extracted immediately from our asymptotic analysis: (i) as the packing fraction increases, the poles move closer to the imaginary axis (cf. [7]); (ii) this tendency also holds for increasing $\gamma_{11}$, or with decreasing temperature, if the stickiness is interpreted as a reciprocal temperature (see, e.g. [26]); and (iii) the distance between the branches is preserved for every given set of parameters.

To be most illustrative, we further simplify above expressions by assuming $\left|c_{1} c_{2}\left(1-\mu^{2}\right)\right| \ll 1$; then the two branches are asymptotically given by

$$
\begin{aligned}
& \mathrm{e}^{-t_{k}^{(1)}} \asymp-\frac{t_{k}^{(1)}}{12 \eta \gamma_{11} c_{1} c_{2}\left(1-\mu^{2}\right)}, \\
& \mathrm{e}^{-t_{k}^{(2)}} \asymp-\frac{t_{k}^{(2)}}{12 \eta \gamma_{11}} .
\end{aligned}
$$

One then sees that variations in the concentration and in $\mu$ leave the branch closer to the imaginary axis practically invariant. Note that for systems I-V in table 1 the assumption $\left|c_{1} c_{2}\left(1-\mu^{2}\right)\right| \ll 1$ is fulfilled.

With these results in mind, we now take a closer look at the pole topology of systems I-VI, which are depicted in figure 1 (omitting the trivial pole at the origin). In systems $\mathrm{I}-\mathrm{V}$ the pole closest to the imaginary axis is a pair of complex conjugates, leading thus to oscillatory decaying behaviour of the PDFs, while in system VI the $g_{i j}(r)$ are exponentially decaying due to the fact that the

Table 1. Parameters for the MSM of the symmetric binary AHS mixtures studied in this contribution; for all systems we assume $R_{1}=1$ and $\eta=0.1$.

\begin{tabular}{lccc}
\hline System & $c_{1}$ & $\gamma_{11}$ & $\mu$ \\
\hline I & 0.10 & 0.3333 & 0.950 \\
II & 0.10 & 0.2727 & 0.950 \\
III & 0.02 & 0.2727 & 0.950 \\
IV & 0.02 & 0.2727 & 0.983 \\
V & 0.02 & 0.2727 & 1.017 \\
VI & 0.40 & 0.9500 & 0.450 \\
\hline
\end{tabular}



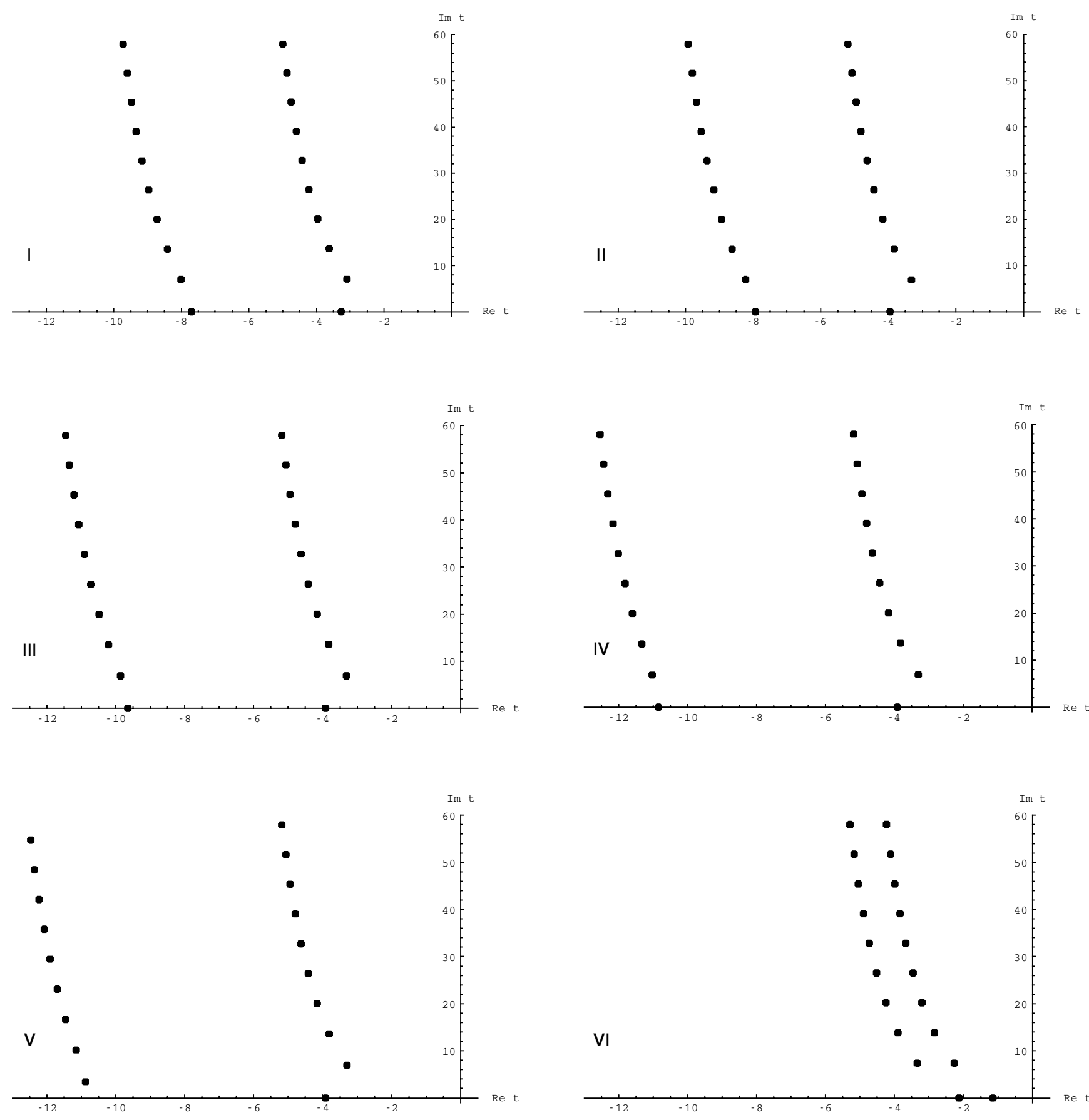

Figure 1. Pole distribution of the MSM $\hat{g}_{i j}(t)$ for symmetric binary AHS mixtures (systems I-VI as hereon and as specified in table 1) in the complex $t$ plane. The search of the poles was restricted to the area $\{-13 \leq \operatorname{Re}(t) \leq 0\} \times\{0 \leq \operatorname{Im}(t) \leq 60\}$.

pole closest to the imaginary axis is real. In particular, the two poles closest to the imaginary axis of systems II$\mathrm{V}$ are pairs of complex conjugates, while in system VI the two poles closest to the imaginary axis are real ones (see also observations in [17]). The parameters of systems $\mathrm{I}-\mathrm{V}$ have been chosen in an effort to visualize their influence on the pole topology: proceeding from one system to the next, we vary only one system parameter, keeping the others fixed. As we go from system I to system II, we decrease $\gamma_{11}$ (or increase the temperature), and hence the branches are shifted to the left in the LH of the $t$ plane, preserving, however, the distance between the branches. We then proceed to system III, by decreasing the concentration $c_{1}$ : the branch closer to the imaginary axis remains (almost) unchanged, while the other one is shifted to the left. In 
going to system IV, we increase $\mu$ to a value slightly below unity, i.e. we approach the one-component case: the left branch is shifted again to the left, while the branch close to the imaginary axis remains again (practically) unaffected. Upon further increasing $\mu$ above 1 (system V), the location of the right hand branch remains unchanged, while we have lost the real pole of the left branch, accompanied by a change in the sequence of the poles of the latter.

\section{Conclusion}

We have investigated the pole topology of the Laplace transform s of the PDFs of a binary AHS mixture within the MSM. This model is complicated enough to include attractive forces, and hence to go beyond hard spheres; on the other hand, it is simple enough to be goverened by a reasonable number of parameters. The pole topology is thus far interesting, as the poles closest to the origin determine how the PDFs approach their asymptotic value, i.e. either exponentially damped or oscillatory decaying. This long range behaviour is also observed in the related non-uniform systems as, e.g. in density profiles. Using the analytical solution of the MSM for AHS mixtures, we are able to give closed expressions that determine the position of the pole branches in the complex $t$ plane. In particular, we could filter out the leading order asymptotic behaviour of these expressions for $|t| \rightarrow \infty$ in the LH of the $t$ plane. Then the dependence of the pole topology on the system parameters becomes transparent. As a by-product of this study we obtain two analytical representations, the SR and the AR, that constitute an accurate and rapidly converging $r$ space method to calculate the PDFs over the entire $r$ range. The expressions presented here are general enough to allow extension of the analysis to multi-component systems.

This research was supported by the Österreichische Forschungsfonds (under Project Nos P13062-TPH and P14371-TPH) and the Wirtschaftskammer Wien. The authors are indebted to Professor R. Evans (Bristol) for useful discussions.

\section{References}

[1] Hansen, J.-P., and McDonald, I. R., 1986, Theory of Simple Liquids, 2nd Edn (New York: Academic Press).

[2] Caccamo, C., 1996, Phys. Rep., 274, 1.

[3] Lebowitz, J. L., and Percus, J. K., 1961, Phys. Rev., 122, 1675.

[4] Ruelle, D., 1969, Statistical Mechanics (New York: Benjamin).

[5] Lebowitz, J. L., and Percus, J. K., 1963, J. math. Phys., 4, 248.

[6] Fisher, M. E., and Widom, B., 1969, J. chem. Phys., 50, 3756.

[7] Abraham, D. B., and Kunz, H., 1977, Phys. Rev. Lett., 39, 1011.

[8] Martynov, G. A., 1992, Fundamental Theory of Liquids: Method of Distribution Functions (Bristol: Adam Hilger).

[9] Evans, R, Henderson., J. R., Hoyle, D. C., Parry, A. O., and Sabeur, Z. A., 1993, Molec. Phys., 80, 755.

[10] Evans, R., Leote de Carvalho, R. J. F., Henderson, J. R., and Hoyle, D. C., 1994, J. chem. Phys., 100, 591.

[11] Henderson, J. R., and Sabeur, Z. A., 1992, J. chem. Phys., 97, 6750.

[12] Leote de Carvalho, R. J. F., Evans, R., Hoyle, D. C., and Henderson, J. R., 1994, J. Phys. Condens. Matter, 6, 9275.

[13] Leote de Carvalho, R. J. F., and Evans, R., 1997, Molec. Phys., 92, 211.

[14] Leote de Carvalho, R. J. F., Evans, R., and Rosenfeld, Y., 1999, Phys. Rev., 59, 1435.

[15] Archer, A. J., and Evans, R., 2001, Phys. Rev. E, 64, 041501

[16] Kahl, G., and Pastore, G., 1991, J. Phys. A, 24, 2995.

[17] Tutschka, C., Kahl, G., and Pastore, G., 2001, Phys. Rev. E, 63, 061110.

[18] Baxter, R. J., 1968, J. chem. Phys., 49, 2770.

[19] Perram, R. J., and Smith, E. R., 1975, Chem. Phys. Lett., 35, 138.

[20] Tutschka, C., and Kahl, G., 1998, J. chem. Phys., 108, 9498.

[21] Baxter, R. J., 1970, J. chem. Phys., 52, 4559.

[22] Blum, L., and Høye, J. S., 1978, J. statist. Phys., 19, 317.

[23] Mier y Terán, L., Corvera, E., and González, A. E., 1989, Phys. Rev. A 39, 371.

[24] Pastore, G., 1988, Molec. Phys., 63, 731.

[25] Waisman, E., and Lebowitz, J. L., 1972, J. chem. Phys., 56, 3086, 3093; R. G. Palmer, and J. D. Weeks, 1973, J. chem. Phys., 58, 4171.

[26] Stell, G., 1991, J. statist. Phys., 63, 1203.

[27] Tutschka, C., unpublished. 